\title{
Golden Ratio and the Fibonacci Series in the Homeopathy Theory and Practice
}

\author{
Bela Tőkés ${ }^{1 *}$ and Attila Tökés ${ }^{2}$ \\ ${ }^{1}$ University of Medicine and Pharmacy in Targu Mures, Romania \\ ${ }^{2}$ Angio-Center Targu Mures, Romania
}

\begin{abstract}
Homeopathy is the medicine of the third millennium. Acoording to homeopathy the only remedy that assure a real remission of te patient is his constitutional chronic one. Potentiation, reached by high dilution and succusion, is the way that eliberates the healing energy of the remedy. In this process are delineated as general laws: the golden ratio and the Fibonacci potency series. The authors explain how the water structure keeps in its "memory" the properties (inclusive the pharmacologcal ones, also) of the active ingredient, and what is the role of the basic dynamisation steps in this mechanism. The Fibonacci potencies series was introduced by Rozencwajg to correctly choose the homeopathic data ("Removing the guesswork from potency selection"). Is demonstrated that succussion might be responsible for creating nano-bubbles. The trituration and succusion process generates shockwaves in a transient localized region of high pressure $(10,000-15,000$ atmospheres) which is powerful enough to trigger fundamental changes in the structure and properties of the carrier media. "Weak Quantum Theory" (a version of Quantum Mechanics) predicts a generalised form of entanglement-a concept established by Schrödinger in 1935. The organism receptors receive and process the information in the remedy according to its state: (1) The raw material for the preparation of a homeopathic remedy is the information matrix. (2) The solvent (water or water-alcohol) employed in the homeopathic preparation is the information carrier: "imprint"- through succession-or "erase"-- through electromagnetic fields-the information contained in it. Homeopathy is now FDA regulated.
\end{abstract}

Keywords: Homeopathy; Golden ratio; Fibonacci series; Potency; Ultradilution; Succussion

Homeopathy in contrast to conventional medicine (allopathy) offers no cure symptoms but shows the way how the patient suffering from the disease may help himself [1-3]. Theoretically it contains even more controversial aspects. The paper outlines a few thoughts on the exact and correct interpretation.

A number of fundamental scientific discoveries of last decades considered homeopathic research milestones are shown below [1-6].

1988: the water structure memory is presented

1990: description is given how the water molecules create structures able to store electromagnetic signals

1991: emphasized that the potentiation creates molecular clusters in water

1999: described that the potentiation changes the properties of water

2001: pointed out that in the water "nanogrouping" are formed

2003: the quantum macro entanglement model is created

2007: found that the succussion creates colloidal nanoparticles

2008: Indicated that homeopathic medicines are stable, clathratetype water structures, of nano-heterogeneous particle dimensions, which can be described by measurable properties

2008: proved that the dilution determines the density of nanobubbles

2009: described that the nano-structures are created by the potentiation

2011: proved that the potentiation of the water molecules creates domains of nanoscale dimensions or nanocrystals
2012: it is shown that the nanoparticles remain stable below the Avogadro limit

Perhaps the scientific recognition of the homeopathy is the fact that the alternative Nobel Prize (Right Livelihood Award) in 1996 was awarded Georgios Vithoulkas, Greek homeopath [4,5,7], for his contribution in the application of classical homeopathy.

Homeopathy is now FDA (U.S. Food and Drug Administration) regulated.

The main operations of the homeopathic practice are the serial high dilution, and the succussion of the (liquid or solid solution) preparation. These operations are jointly termed potentiation. Not enough dilution required succussion, too.

A serial dilution proposed by Hahnemann [8] and other schools [9-11], can be feasible in various ratios, of which the most usual are the decimal (D: $1 / 10$, and this continued for a further $1 / 10$ with dilution on every new sequence term) and the centesimal (C: same, but now 1/100 scale serial dilutions) technique. For example, using the latter series, the molar concentrations will be reduced in the following ratios: $10^{-2}$, $10^{-4}, 10^{-6}, 10^{-8}, 10^{-10} \mathrm{M}$, etc. Here, for the sake of simplicity, it is assumed that we started from a $1 \mathrm{M}$ stock solution ("mother tincture"). Highest

*Corresponding author: Bela Tőkés, Professor Emeritus, University of Medicine and Pharmacy in Targu Mures, Romania, Tel: 40365405 794; E-mail: belatokes@yahoo.com

Received December 05, 2013; Accepted Janaury 09, 2014; Published Janaury 11, 2014

Citation: Tökés B, Tőkés A (2014) Golden Ratio and the Fibonacci Series in the Homeopathy Theory and Practice. Altern Integ Med 3: 149. doi:10.4172/23275162.1000149

Copyright: (ㅇ 2014 Tökés B, et al. This is an open-access article distributed under the terms of the Creative Commons Attribution License, which permits unrestricted use, distribution, and reproduction in any medium, provided the original author and source are credited. 
Citation: Tökés B, Tökés A (2014) Golden Ratio and the Fibonacci Series in the Homeopathy Theory and Practice. Altern Integ Med 3: 149. doi:10.4172/2327-5162.1000149

Page 2 of 7

concentrations are already of the solubility rare, but if the dilution is desired, it would be meaningless. Easy to see that solution of $12 \mathrm{C}$ no longer contains a single molecule. In case of homeopathy the main point of attack is connected with the increasingly high dilution. Question is usually that why can act a solution, wherein the active substance is not present nor in a single molecule? At first glance this appears to be well founded objection [12-14]. Really it does not contain a single molecule of the active substance, either, of course, on average, if the fluctuations are taken into account. Even Hahnemann himself felt that in ordinary causal relationships cannot rely on. He assumed that the solvent (usually water or alcohol-water mixture) preserves the memory of the properties ("fingerprints") of the substance (pharmacological ones too) and passes them on your body (of course, receptors does not imply the existence of). It is worth recalling that when Benveniste (1988) also "trusted" the transmission of information to the memory of water [15-18], his colleagues mocked him, but they also, later, when it did not have a Nobel Prize Award to Benveniste excluded, flattered their former irony target.

It is interesting that the "memory effect" more often is detected in different areas, over time. e.g. in electrode reactions $[19,20]$ the deposition potential depends also on if the depolarizer molecule is directly introduced into the reaction space, or as a product of a previous process it "born" in the electrode force field, and its spatial orientation and further fate are predetermined. In the latter case, the intermediate product already includes some information, they define the character of the next stages, and which are absent in the case of compounds introduced from outside. The electrode is actually the source of electrons entering in and out of depolarizer agent. The transmission of information to the electrode/electron system is easy to see. It is worth noting: the memory effect is also established by a field of force (energy transfer).

Also known the role of the structure of liquid water forming hydrogen bridge bonds $[21,22]$. The existence of molecule association groups were already known in the first half of the last century. Examining the hydrogen bonds in the context of an universal relation comes in sight the law of the golden ratio (golden-mean) [23]. It describes a (very often recognizable and considered as universal) relationship according to which from two quantities ( $a$ and $b, a>b$ ) the larger one is proportional to the smaller parts such as the lesser extent to their difference, that is, $b$ is the geometric mean of theses latter quantities:

$\underbrace{a b b}_{a+b}$

$$
\frac{a}{b}=\frac{b}{a-b}
$$

that is

$$
b^{2}=a \cdot(a-b)
$$

The golden ratio numerically is described by an irrational number: $\Phi \approx 1$.618. Its inverse is $\varphi \approx 0.618$. Figure 1 shows the case of the hydrogen bridge

The hydrogen bridges present a tetrahedral space structure of water molecules, with a relatively rigid hexagonal conformation. This means a more or less energetically stable state can be developed, separated by energy barriers. Due to the relatively low activation energies (several $\mathrm{kJ} / \mathrm{mol}$ ) quasi-continuous transition is achieved between the stable and

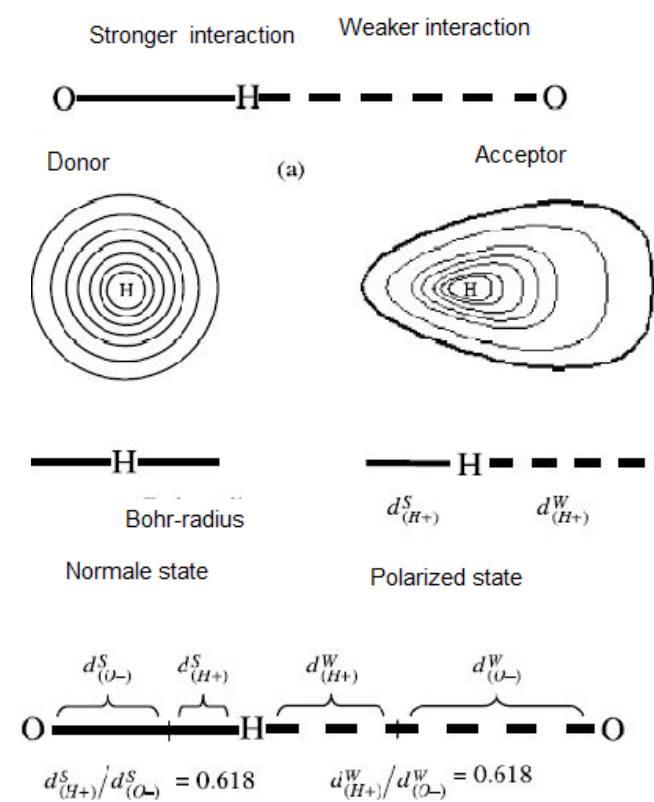

(c)

Figure 1: The golden ratio at the $\mathrm{H}$-bond.
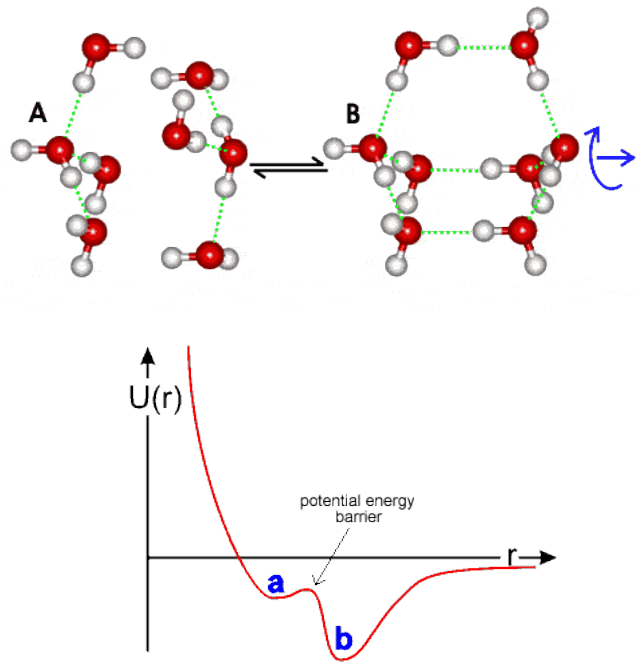

Figure 2: The water conformation and the potential energy variation.

metastable states. The golden mean may immediately be observed in Figure 2. Certainly other examples can be cited as well.

According to the above, the water conformational associations are determined by their related energy field.. Energy fields can also be created by molecules, ions, free radicals-actually also by molecules of active pharmaceutical ingredients, and the independent water structures themselves can interact with other molecules. In order to exert an effect the active constituents are linked to proteins (receptors). The force fields generated by the active particles and the corresponding water cluster structures play a decisive role in the behavior of the partner molecules.

Emoto [http://www.is-masaru-emoto-for-real.com] has shown 
Citation: Tökés B, Tökés A (2014) Golden Ratio and the Fibonacci Series in the Homeopathy Theory and Practice. Altern Integ Med 3: 149. doi:10.4172/2327-5162.1000149

that water ice-in a globally ordered crystalline structure-will create different geometric shapes, depending on the crystallization conditions. These conditions are influenced by solutes, by different energy fields, -including music-, just as by mental control. Some apparently absurd example may be quoted in Figure 3.

The Kirlian spectra (auras) of water in Figure 4 and the dissolved substances (drugs) [24] may lead to similar conclusions. It seems evident the water models different structures in various energy fields and conditions, respectively various water structures generate different energy fields.

Chaplin in his critical analysis [25-27] showed the stability of the clusters increases with the dilution. This interpretation is in accordance with the Arndt Schulz's rule (1888), as substances (drugs) which are toxic in large amounts present a curative effect when diluted.

As it was already noticed, the medium is often a mixture of water and ethanol. According to a nice story an international team observed differences in hydrogen-bonding strength in vodkas using H-NMR, FT-IR, and Raman spectroscopy. It was suggested differences in taste are given by clathrate structure. The story goes, that the Nobel Prize winner Linus Pauling believed the clathrates give alcohol a narcotic effect.

It is to emphasize, in the potentiation the shaking has a decisive effect $[3,11,25,26]$. This explanation should lay in the instant pressure effect of ten or fifteen thousand atmospheres causing the structural changes the in water associations (respectively in other molecular clusters). It seems, it is a "necessary and sufficient" condition. The dilution itself

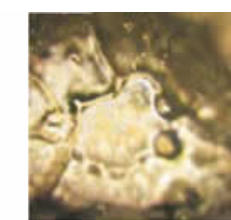

Water Molecule, Before Offering a Praye

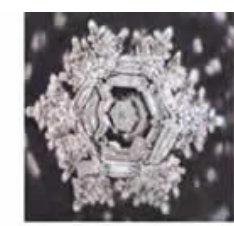

Water Molecule, After Offering a Prayer

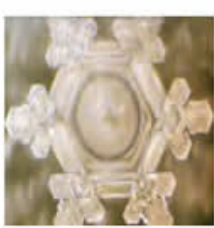

Thank You

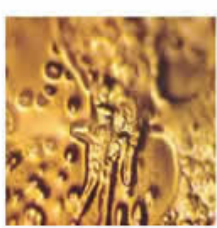

You Make Me Sick I Will Kill You

Figure 3: Water crystals images.

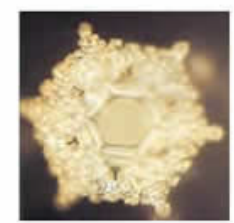

Love and Appreciation

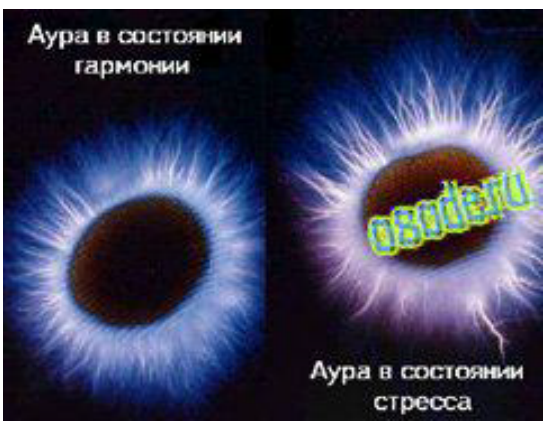

Figure 4: Kirlian's auras in harmonic and stressed states.

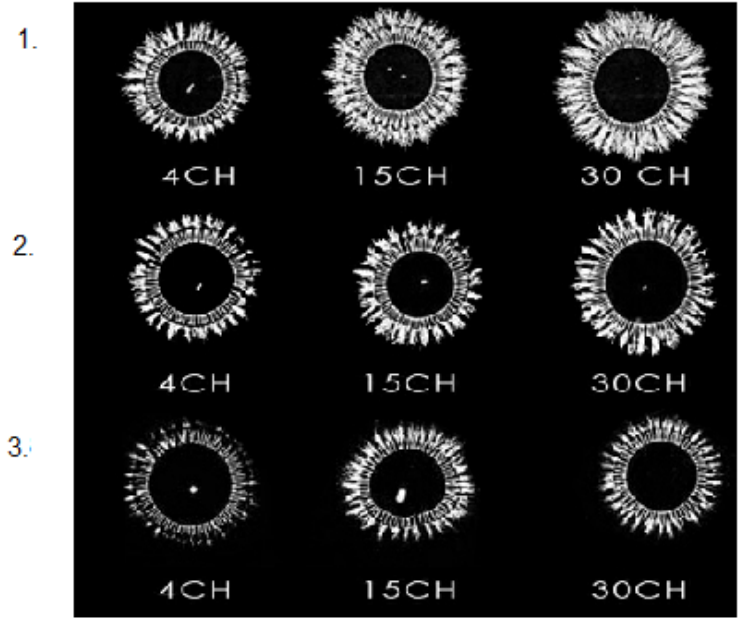

Figure 5: Kirlian's auras in different homeopatic conditions:

1. seriea: 4C-15C-30C dilutions with manual succusion

2. series: 4C-15C-30C dilutions with mechanic succusion

3. series: 4C-15C-30C solutions, no succusion

is necessary for storaging and transmission of information. In fact the two operations together satisfy the "necessary and sufficient condition" (Figure 5).

The same effect as by a homeopathic drug may also be achieved if the energy is transmitted by other means to the (biological) system, (e.g. by light-interference devices).

Energy transmitted by the succussion is accumulated and causes fast mechano-chemical effects provoked by free radicals and other mechanisms (it occurs in femtosecond of time, $1 \mathrm{fs}=10^{-15} \mathrm{~s}$ ) generating different structures (clusters, domains).

Between the ultrasonic chemistry [28] and the succussion an analogy can be perceived The ultrasound can be divided into active and passive range, which-by convention-the power is considered passive under $1 \mathrm{~W} / \mathrm{cm}^{2}\left(10 \mathrm{~kW} / \mathrm{m}^{2}\right)$, and active above this value. Passive ultrasounds are used for testing materials, while the active ones are applied to alter their properties.

In the 1930s German chemists observed that dispersed substances in aqueous solution will react when exposed to ultrasonic effect even if normally they would not react with one another. The explanation is that the ultrasounds create bubbles, and by collapse these bubbles-due to the Casimir effect-release an impact with so much energy as a significant local heating occurs (The reaction space may be heated to thousands of degrees Kelvin). This sonochemical effect was soon forgotten, because the practical application proved to be too expensive [29]. However in the 1990s, as more advanced measurement techniques were available, it was noticed that the collapsing bubbles sometimes generate an energy with several orders of magnitude higher than expected by normal physical reasons Due to the high energy the collapsing bubbles can flash, so this phenomenon is also known as sonoluminescence. After a long time of searching for an explanation, the answer was finally found by the Nobel Prize-winner physicist Julian Schwinger as that the vacuum energy is responsible for the significant temperature raise [30].

The behavior of homeopathic preparations may be explaind based on the quantum entanglement phenomenon [1,23,31,32]. This concept, described in 1935 by Erwin Schrödinger, the Austrian Nobel laureate, was originally known in the 1930s, as Einstein-Podolsky- 
Rosen paradox. The entangled particles are not separate units, but they should be seen as a complete system [http:/www.newscientist.com/ article/mg20927952.900scorn-over-claim-of-teleported-dna.html].

Nobel Prize-winner Montaignier et al. [33] has shown that organized water clusters (nanoparticles, domains) are able to transmit electromagnetic signals between each other and with the outside world. This is a special case of resonance, and satisfies the requirements of quantum entanglement. The phenomenon has been modelled in 2003. Certainly the quantum theory has to be applied to the bonds, the electrons involved in bonding must be characterized by the particlewave duality, reflecting the principle of Heisenberg's uncertainty, and the bondones have to present quantum nature [23]. In fact all these theories, show a strong correlation with the rule of golden ratio.

Concerning the Montagnier experiments, J. Reimers, says, "If the results are correct, these would be the most significant experiments performed in the past 90 years, demanding re-evaluation of the whole conceptual framework of modern chemistry" [ http://en.wikipedia.org/ wiki/Luc_Montagnier].

Montagnier produced biologically active "aqueous nanostructures" like in homeopathic medicine, using the serial agitated dilution in water. His research on detection of electromagnetic signals in the plasma from patients with Alzheimer's and Parkinson's disease, multiple sclerosis and rheumatoid arthritis confirms what the homeopaths have been telling the scientific world for many years $[1,2,11,30,33-36]$.

Montagnier found that in the stimulation of the dilute an electromagnetic background of very low frequency was essential. The background was either produced by natural sources (the Schumann resonances which start at $7.83 \mathrm{~Hz}$ ) or by artificial ones. Homeopaths considered these studies as a further proof, that homeopathic remedies have distinct supramolecular structural features emitting electromagnetic signals that can affect biological entities. It is also providing further insight into the physics of homeopathy.

Montagnier have proved the major point of contention in favor of homeopathy, respectively the memory of water, which is biologically specific.

The sinusoidal wave is a common graph for results in almost all homeopathic studies, no matter are they physical, in vivo or in vitro. As dilutions increase changes in amplitude and frequency may occure. The most commonly used dilutions in available homeopathic remedies OTC are 6C, 12C and 30C. A keen mathematical eye might spot what the relationship is between those three numbers: they fall upon an advanced Fibonacci scale, mathematically defining the spiral (Figure 6).

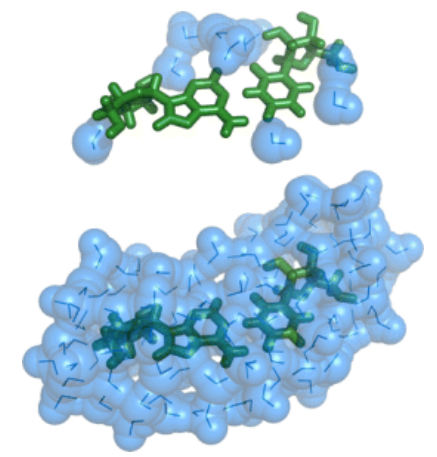

Figure 6: The clathrate, homeopathy's missing link. RNA with solvation shell.

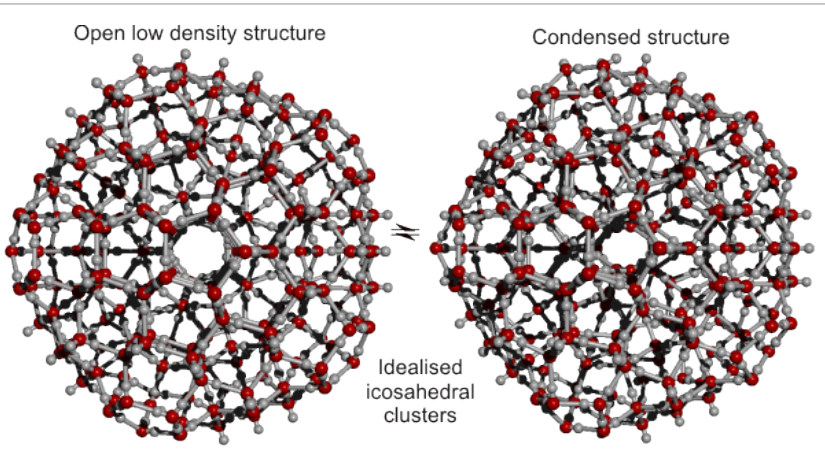

Figure 7: Structure variations and quantum cooperation in water

Richard Bartlett [31] introduced the energy concept describing the state of being (using an energy matrix) and the concept how to control the disease. In his view, all energy species, including the human energy field-known as the morphogenetic field-are under concious control. Actually the forerunner of this idea is the Jungian collective unconsciousness. The morphogenetic field is the blueprint of life-the source of information for DNA.

Physicists have only recently demonstrate that an electron or a photon may appear in multiple spatial locations at a time, which is an unusual perception and rather exciting in itself. It might also offer a new dimension to notions regarding teleporting. Perhaps even more astonishing is that any change in the status of the particle pairs of "twins" can lead to a change-wihout delays of any kind and regardless of distance (the speed of light is not a limit). Now, in the extremely noisy environment the quantum entanglement can be experienced (eventually even investigated) in a biological medium, at room temperature. These findings put a new light on understanding the coordinated thinking and action of the twins from a singel ovum.

Going back to the quantified state of the water domains, Mae-Wan Ho [http://www.i-sis.org.uk/QuantumJazz.php] suggested in 1990, that the water quantum-coherence is the life itself (Life is Water's Quantum Jazz). The quantum co-operativity is manifested by the $\mathrm{H}$-bridges formed between the water molecules, i.e. the interaction between the local units conducting to global effects resulting in the formation of supramolecular clusters. The icosahedral shapes of $\left(\mathrm{H}_{2} \mathrm{O}\right)_{280}$ are an example. This assumption was later proved by quantum chemical calculations as well (Figure 7).

The concept of quantum entanglement and interactions (and increasingly its practice) is consistent with the torsional waves of Tesla (1900), [http://www.fractalfield.com/fractalalchemy] later extended ( 1970) by Kozyrev to various systems (including the structure of the water domains).

The Fibonacci series should also express (besides the golden ratio), an universal law (applicable to water domains as well) and with a closely related other natural law. This is originally described by a specific progression, wherein each member is the sum of the two previous ones: (0), $1,1,2,3,5,8,13,21,34,55,89,144,233$, etc. Later, more general series (Lucas, Binet, etc.) were also developed with members deducted from Pascal's triangle (Figure 8).

The formula and the figures illustrate the method of calculation, the relationship with the golden ratio, their graphic illustration and derivation from the Pascal's triangle. Taking into account as the golden ratio itself may be considered a progression wherein any of the members is the geometric mean of the previous and the next members 

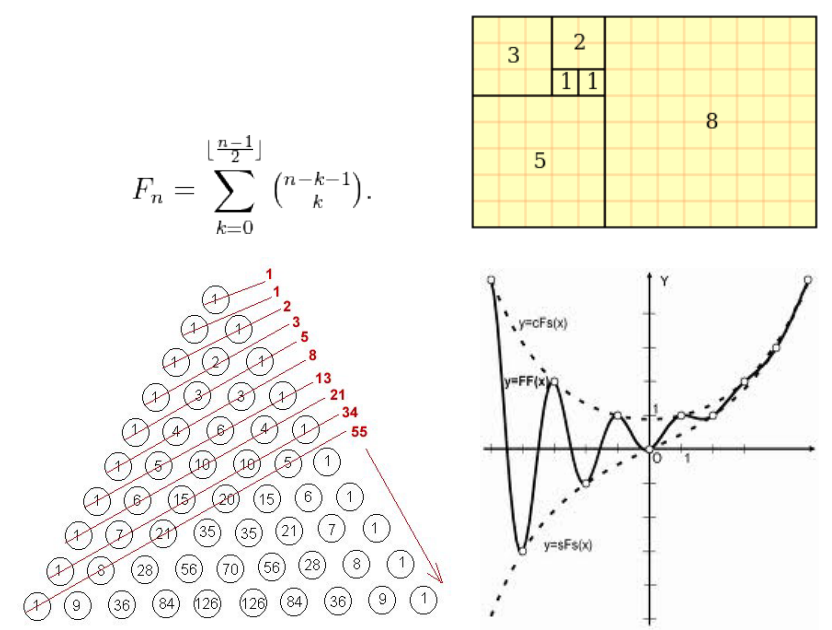

Figure 8: Relations between the golden rate and the Fibonacci series.

\begin{tabular}{|c|c|c|c|}
\hline $\begin{array}{c}\text { Fibonacci } \\
\text { Series }\end{array}$ & $\begin{array}{c}\text { Starting potency (3C) } \\
\text { and the dilution } \\
\text { series }\end{array}$ & $\begin{array}{c}\text { Starting potency (5C) } \\
\text { and the dilution } \\
\text { series }\end{array}$ & $\begin{array}{c}\text { Starting potency 8C) } \\
\text { and the dilution } \\
\text { series }\end{array}$ \\
\hline $\mathbf{1}$ & $3 \mathrm{C}$ & $5 \mathrm{C}$ & $8 \mathrm{C}$ \\
\hline $\mathbf{5}$ & $15 \mathrm{C}$ & - & - \\
\hline $\mathbf{8}$ & $120 \mathrm{C}$ & $40 \mathrm{C}$ & - \\
\hline $\mathbf{1 3}$ & $1.5 \mathrm{M}$ & $520 \mathrm{C}$ & $104 \mathrm{C}$ \\
\hline $\mathbf{2 1}$ & $33 \mathrm{M}$ & $11 \mathrm{M}$ & $2 \mathrm{M}$ \\
\hline $\mathbf{3 4}$ & $1 \mathrm{MM}$ & $370 \mathrm{M}$ & $74 \mathrm{M}$ \\
\hline $\mathbf{5 5}$ & $61 \mathrm{MM}$ & $20 \mathrm{MM}$ & $4 \mathrm{MM}$ \\
\hline $\mathbf{8 9}$ & $5.5 \mathrm{MMM}$ & $2 \mathrm{MMM}$ & $363 \mathrm{MM}$ \\
\hline $\mathbf{1 4 4}$ & $78 \mathrm{MMM}$ & $262 \mathrm{MMM}$ & $52 \mathrm{MMM}$ \\
\hline $\mathbf{2 3 3}$ & $180 \mathrm{MMMM}$ & $61 \mathrm{MMMM}$ & $12 \mathrm{MMMM}$ \\
\hline
\end{tabular}

Table 1: Often used potency scales as function of Fibonacci series.

(in the same time the coefficient $\mathrm{a} / \mathrm{b}=\Phi \approx 1.618$ that is $\varphi \approx 0.618$, universal constant expressed by an irrational number). Compared to the Fibonacci terms, it will be observed that the numbers of the two series coincide. At the beginning the graphical image is a waveform curve with decreasing amplitudes. The oscillation is also a common (perhaps universal) natural phenomenon, manifesting, in the biological systems, both in organic and inorganic world (e.g. in chemical processes).

Fibonacci series seem to be omnipresent. It can be observed in nature, as the mathematical proportion of shapes of flowers, cacti, vegetables like Brassica, Pineapple, Nautilus Shell, Butterfly, inner and external human ears etc. It is also used in many mathematical formulas for accounting and trading. It is even present in art and music. The rhythm found in nature led to use the same one in homeopathic potencies and such series of centecimal potencies are called the Fibonacci Potencies.

Rozencwajg examined the logic between the different potency scales (The title of his paper is significant: "Removing the Guesswork from potency selection"). He observed initially a linearity between the series, but the nature of the relationships is rarely linear. The regularity became more evident when he reached the examination of the golden ratio and Fibonacci series [http://hpathy.com/homeopathy-papers/ the-fibonacci-potencies-series-update-discussion-and-conclusions].

For practical homeopathic purposes, the Fibonacci series begin with the lowest potencies. On a centesimal scale, depending on the agent, these are $3 \mathrm{C}$ or $5 \mathrm{C}$. Starting with $3 \mathrm{C}$, the following members of the series are $5 \mathrm{C}$, then $8 \mathrm{C}(3+5)$, followed by $13 \mathrm{C}(5+8), 31 \mathrm{C}, 34 \mathrm{C}, 55 \mathrm{C}$, 89C, $233 \mathrm{C}$ etc.

Rozencvaig feared that these relatively low potencies do not produce the desired effect. Howevert he found that they grow further exponentially and lead to excellent results. For example, starting with a 5C potential dose and continuing later the administration with the same medication of $8 \mathrm{C}$ potency, the effect was equivalent with $40 \mathrm{C}(5 \times 8)$, and if the next dose was 13C, their overall effect became equivalent to 520C $(13 \times 40)$, a.s.o.. Looking in more details on the evolution of the frequently used initial potencies (Table 1) seems to be useful. Starting potencies are considered: $3 \mathrm{C}, 5 \mathrm{C}$ or $8 \mathrm{C}$.

3C: This is the so-called "low potency", the starter potency for non-toxic plants and non-toxic materials. Its actual concentration-or rather dilution-is one part per million (1 ppm), still detectable with regular laboratory techniques. Without taking into account the factor of dynamisation, $1 \mathrm{ppm}$ still has physiological effects and therefore may not be used with toxic substances, poisons, venoms etc.

It is to be emphasized these potencies are not a simple succession of weird ones, but they have a multiplying, exponential effect, allowing to reach extraordinary high potency levels which otherwise should be unattainable.

5C: This level of dilution is equivalent to ten parts per billion (10 $\mathrm{ppb}$ ). $5 \mathrm{C}$ is used as the starter potency for toxic plants, poisons, metals and minerals in general. Some of these are not available in a lower dilution.

\section{C: It is a starting potency for the nosodes.}

Currently, preparations of the Fibonacci-type homeopathic potency series are produceded only by a few pharmaceutical companies, often up to 33C, The customized values can be obtained by anyone "at home" from the accessible preparations of intermediate potency.

As the mother tincture is more toxic, it is preferable to use dilutions until the probability of an atom or molecule of drug entering the patient body during the treatment is reduced close to zero. No matter what the dilution is, the probability never drops to zero can only approach it. It is understandable, the higher the dilution, the more time, energy, carrier agent are necessary for the preparation of the drug solution. To improve the situation, the active ingredient will only be diluted until the information is transcribed. For this purpose adequate instruments are necessary for measuring or-at least to estimate-the information content. When these are not available the only possibility remains optimizing the succussion.

Usually drug molecules have been removed from the information carrier substrate by dilution. Alternatively, a variety of careful screening procedures may be used. These may be: molecular sieve, osmosis filter, fine centrifugation or sedimentation. The point is that the screening process may not compromise the information content of the carrier.

Carrier of distilled water is recommended as the presence of dissolved minerals change the structure clusters of the water molecules. The cleaner-physically and "informatically"-the carrier is, the noiseless will be the drug made of it.

For the transcription of information two ways are considered:

- Primary (direct) transcription encountering a drug particle to a carrier molecule. 
Citation: Tökés B, Tökés A (2014) Golden Ratio and the Fibonacci Series in the Homeopathy Theory and Practice. Altern Integ Med 3: 149. doi:10.4172/2327-5162.1000149

Page 6 of 7

- Secondary or repeated transcription which encounters a transcribed substrate molecule with a pure one.

As it might be expected the primary way is more efficient. The first potentiation has the best effect, and the least number of successions is required. The more diluted the drug (or the higher dilution is used), the more succussions are necessary for a successful transcription. (A single drug molecule must collide with several carrier molecules for a primary transcription). It is necessary to insert a rest between each succession for satisfactory mixing the molecules. Before the succession it is efficient to apply a good mixing of the mother tincture with the carrier.

In case the symptoms induced by the active ingredient are more similar to those of the disease, a more dilute agent must be used. The reason is that the "injected" information will stimulate the body, but in the absence of the toxic drug the antibodies produced by the immune system, cannot attack its molecules, and they will concentrate on the disease carrier (the patient body). Essentially, the body was "misguided" and it is switched over to natural control.

Low dilutions of substances are effective in physical sicknesses whereas high ones in the psychlogic ones as well. The reason lies in the information content. The psyche and the body are in permanent information contact and interact with each other. The principle of "sound mind in a sound body" applies. The sick soul makes sick its body and the sick patient's body cripples the soul. Improving the general feeling of body can improve the state of mind. This dynamic feedback and self-perpetuating, self-protective process may interfere with various substances (like toxins, drugs, aphrodisiacs a.o.) or psychological factors (learning, habituation, hypnosis, suggestion, meditation, etc) just as energetic actions as well (acupuncture, earth radiation, etc.). The highly diluted substances have to be administered rarely, as they maintain the stimulatory effect for a longer time. Potentiation is done manually with 10 succussions at each stage.

In summary, once the drug is selected; the most important operation is its potentiation. Rozencwajg, a sailant personality of modern homeopathy, an excellent knowner and tiller of acupuncture, homeopathy, TCM (Traditional Chinese Medicine), food sciences, herbs, aromatherapy, natural medicine, Reiki, and other areas, developed more potency scales [37]. These studies, highlighting the need for homeopathy, launched a tremendous progress-on the bases of the Fibonacci potency scale-both from theoretical and practical (operational) point of view.

From all those outlined it seems clear (and that's only the tip of the iceberg !) that homeopathy is studied by the most prominent experts and huge volume of facts and results are processed (a real MountBlac of data). Modern research methods and high-tech achievements have been launched in the battle. It's enough to notice the modern spectrometry, various forms of modern diffraction techniques, the thermometric, chemical equipment, electrical, magnetic, and optical devices.

\section{References}

1. Bellavite P, Signorini A (2002) The Emerging Science of Homeopathy. North Atlantic Book, Berkeley.

2. Dobrescu D (2011) Farmacologie Homeopata Ecologica. Farmacologie generala. (3rdedn). Universitara, Bucureti.

3. Ernst E (1997) Homoeopathy: past, present and future. Br J Clin Pharmacol 44: $435-437$

4. Vithoulkas G (1880) The Science of Homeopathy. Grove Press, New York.
5. Vithoulkas G (2000) Homeopathy: Medicine for the New Millennium. IACH California Hypnosis Institute.

6. Ullman D (1991) Discovering Homeopathy: Medicine for the 21st Century. North Atlantic Books, Berkely.

7. Vithoulkas G (1892) A New Model for Health and Disease. North Atlantic Books, Berkeley.

8. Hahnemann S (1960) Organon of medicine. (6thedn). Calcutta: M Bhattacharyya \& Co.

9. Elia V, Niccoli M (1999) Thermodynamics of extremely diluted aqueous solutions. Ann N Y Acad Sci 879: 241-248.

10. Khuda-Bukhsh AR (2003) Towards understanding molecular mechanisms of action of homeopathic drugs: An overview. Mol Cell Biochem 253: 339-345.

11. Khuda-Bukhsh AR, Pathak S (2008) Homeopathic drug discovery: theory update and methodological aspect. Expert Opin Drug Discov 3: 979-990.

12. Anagnostatos GS, Pissis P, Viras K, Soutzidou M (1998) Theory and experiments on high dilutions. In: Homoeopathy-a critical appraisal Butterworth-Heinemann, Reed Educational and Professional Publishers Ltd.

13. Berkowitz CD (1994) Homeopathy: keeping an open mind. Lancet 344: 701702 .

14. Berwin TH (1995) What's wrong with complementary medicine? The Sceptic 8: 6-9.

15. Benveniste J, Ducot B, Spira A (1994) Memory of water revisited. Nature 370 322.

16. Benveniste J (1994) Further Biological Effects Induced by Ultra High Dilutions: Inhibition by a Magnetic Field. Kluwe Academic Press, Dordrecht.

17. Benveniste J, Aïssa J, Guillonnet D (1999) The molecular signal is not functional in the absence of "informed" water, FASEB Journal 13: A163.

18. Benveniste J (2005) Ma vérité sur la 'mémoire de l'eau' (Préface du professeur Brian D. Josephson, Prix Nobel de Physic). Albin Michel, Paris.

19. Donáth-Nagy G, Buchwald P, Vancea S, Croitoru M, Tokés B (2008) The quantitative characterization of free radical sources and traps by electromigration applications. J Biochem Biophys Methods 70: 1317-1323.

20. Tókés B, Suciu G, Nagy G (2002) Interdependent chemical-electrochemical steps in retrometabolism-based drug and safer chemical design. Pharmazie 57: $122-126$.

21. Elia V, Napoli E, Germano R (2007) The 'Memory of Water': an almost deciphered enigma. Dissipative structures in extremely dilute aqueous solutions. Homeopathy 96: 163-169.

22. Prokop O, Hopff W (1992) Erklarung zur Homöopathie. Dtsh Apotheker Zeitung 132: $1630-1632$

23. Putz M V (2012) Valence atom with bohmian quantum potential: the golden ratio approach. Chemistry Central Journal 6: 135-157.

24. Opalinski $J$ (1979) Kirlian-type images and the transport of thin-film materials in high-voltage corona discharges. Journal of Applied Physics 50: 498-504.

25. Chaplin MF (2000) A proposal for the structuring of water. Biophys Chem 83 211-221.

26. Chaplin MF (2007) The Memory of Water: an overview. Homeopathy 96: 143 150.

27. Chaplin MF (2008) Reply to Comment on 'The memory of water; an overview'. Homeopathy $97: 43-44$

28. Silvo M, Arnaldo P (1990) Ultrasonographic study of homeopathic solutions. $\mathrm{Br}$ Homeopathic J 179: 212

29. Bordag M, Mohideen U, Mostepanenko VM (2001) New Developments in the Casimir Effect. Phys Rep 353: 1-205.

30. Jack Ng Y (1996) Julian Schwinger J The Physicist, the Teacher, and the Man Singapore: World Scientific.

31. Bartlett R (1998) Matrix Energetics: The Science and Art of Transformation, Ed. Adevar Divin, Brasov.

32. Feynman RP (1985) Introduction in QED: the strange theory of light and matter Princeton University Press, Princeton, NJ 1-20 
Citation: Tőkés B, Tőkés A (2014) Golden Ratio and the Fibonacci Series in the Homeopathy Theory and Practice. Altern Integ Med 3: 149. doi:10.4172/2327-5162.1000149

Page 7 of 7

33. Montagnier L, Aïssa J, Ferris S, Montagnier JL, Lavallée C (2009) Electromagnetic signals are produced by aqueous nanostructures derived from bacterial DNA sequences. Interdiscip Sci 1: 81-90.

34. Benveniste J (1993) Molecular signaling at high dilution or by means of electronic circuitry. Journal of Immunology 150: 146A.
35. Khuda-Bukhsh AR (1986) Some homeopathic drugs as radio-protective agents in X-irradiated mice. Perspectives in Cytology and Genetics 5: 407-412.

36. Benveniste J (1994) Transfer of the molecular signal by electronic amplification, FASEB Journa 8: A398.

37. Rozencwajg J (2009) The Potency: Advanced Prescribing in Homeopathy Emryss Haarlem. 